\title{
ERAS: medicina basada en evidencia, medicina basada en resultados, medicina basada en valor
}

\author{
ERAS: evidence-based medicine, results-based medicine, value-based medicine \\ ERAS: medicina baseada em evidências, medicina baseada em resultados, \\ medicina baseada em valor
}

Ángela Navas Camacho*

https://doi.org/10.35454/rncm.v3n1.013

Desde mediados del siglo XIX los avances en las técnicas anestésicas, el desarrollo de los antibióticos en la primera mitad del siglo XX y el ascenso ininterrumpido en la generación de conocimiento en farmacología, el desarrollo de la nutrición parenteral, el cuidado intensivo y nuevas tecnologías en instrumentos quirúrgicos y ópticos con la carrera militar y espacial han posicionado a la cirugía como un componente esencial en la atención en salud, convirtiéndose en herramienta de primera línea para el manejo de los pacientes con patologías específicas.

Cada uno de estos avances ha obligado a una rápida adaptación del personal de salud en la adopción de estrategias que eran impensables para los cirujanos décadas atrás y que hoy hacen parte del estándar de práctica. Este cambio ha ido acompañado de un explosivo aumento del número de procedimientos quirúrgicos, que para el año 2012 alcanzó los 310 millones de cirugías ${ }^{(1)}$. Sin embargo, este incremento está acompañado de complicaciones en $26,8 \%$ de los pacientes y mortalidad en $5 \%$. Cerca de $50 \%$ de los eventos adversos de pacientes hospitalizados se relacionan con la atención quirúrgica y al menos la mitad de ellos se considera prevenible ${ }^{(2)}$.

La atención ha tenido como centro la figura del cirujano que ha demostrado la capacidad de desarrollar habilidades extraordinarias que no necesariamente se ven reflejadas en la disminución de complicaciones, pero que sumadas a la introducción de tecnologías necesarias lleva año tras año a un incremento en los costos. Todo esto ha llevado a la aparición de políticas en salud y guías clínicas para orientar la utilización de los recursos limi-

* angelamarianavas@gmail.com tados, que infortunadamente no aumentan en la misma proporción que los gastos, obligando a tener esquemas estructurados para la toma de decisiones basados en la mejor evidencia disponible ${ }^{(3)}$.

El objetivo de la cirugía moderna es ofrecer una atención de calidad, evaluando las nuevas tecnologías y protocolos no solo en términos de evidencia sino también de costo-efectividad, minimización de costos y costo/beneficio, utilizando modelos económicos en salud basados en datos estadísticos, para evaluar el impacto financiero de una patología o un tratamiento propuesto. Es así como la atención con calidad deja en el centro al paciente rodeado de un equipo multidisciplinario que utiliza la mejor evidencia, siempre de la mano del mejor juicio clínico y pensamiento crítico, obligando a que los cirujanos seamos medidos y medibles.

Para complementar es necesario vincular al grupo de gestión de calidad en salud. Inicialmente, y debido a nuestra manera tradicional de trabajo, consideramos que la auditoría y la gestión llegaban para "enredar" el trabajo de los clínicos, pero contrario a esa primera impresión nos han permitido estructurar los procesos de trabajo, evaluando la eficacia mediante indicadores de calidad y planteamiento de oportunidades de mejora.

Lo anterior hace que Latinoamérica sea el escenario perfecto para la adopción de estrategias en salud que mejoren los desenlaces y sean costo-efectivas, idealmente como políticas de salud en los ámbitos gubernamental e institucional. En la gran mayoría de nuestros países la práctica médica es de alta calidad y se cuenta con tecnología de punta. Sumado a esto, los procesos de acreditación en salud van estimulando una atención hospitalaria de calidad. Los programas de salud que 
combinan la medicina basada en evidencia, la medicina basada en resultados y la medicina basada en valor son una buena estrategia cuando los recursos para inversión son limitados.

Los programas de rehabilitación multimodal en pacientes quirúrgicos, en los cuales se destaca ERAS, han logrado un poder transformador en la práctica médica, creados al inicio para cirugía colorrectal y actualmente extendiéndose a otros tipos de cirugía, utilizan múltiples estrategias basadas en la evidencia, incluidas en un protocolo multidisciplinario mostrando mejoras en los desenlaces de los pacientes (disminución de complicaciones postoperatorias, reducción de estancia hospitalaria y reducción de costos de la atención). La adopción de estos programas ha permitido tener un mejor control de los procesos quirúrgicos, protocolizar los manejos y unificar conductas respecto al uso de medicamentos, insumos y tecnologías, tener control de la calidad de la atención y mejor utilización de los recursos de salud.

El término ERAS surgió de un grupo de cirujanos europeos que en el año 2001 creó el grupo de estudio con el fin de desarrollar unas vías de cuidado perioperatorio óptimo, basados en los trabajos realizados por el grupo del doctor Henrik Kehlet desde 1995, usando la mejor literatura y evidencia disponible asociado a un adecuado registro de la adherencia a cada una de las estrategias, evaluando así los progresos o las necesidades de cambio. En el año 2010 nace la sociedad ERAS ${ }^{(4)}$ que conocemos ahora como multiprofesional y multidisciplinaria que vela por el desarrollo, la educación y la investigación en el cuidado perioperatorio. Durante estos 18 años de trabajo, el número de instituciones ERAS en el mundo ha ido en ascenso, en el año 2015 se otorgó un certificado al Hospital Italiano de Buenos Aires que lo acredita como primera institución ERAS en Latinoamérica, y ahora con presencia en Uruguay, Brasil, México y Colombia.

En este número de la Revista, contamos con la participación del Hospital Santa Casa de Porto Alegre que nos muestra su proceso de implementación del protocolo ERAS y los resultados en mejora de la estancia hospitalaria y reducción de complicaciones. El grupo del Hospital Italiano de Buenos Aires nos presenta su experiencia y lo que se ha logrado hasta el momento en ERAS Latinoamérica como una muestra de la capacidad para desarrollar investigación y programas de calidad.

A lo largo de estos años, diversos grupos han creado otros programas de Recuperación en cirugía como el Projeto ACERTO Brasil, que el doctor Aguilar-Nascimento presenta en un artículo de revisión en este número.
La intervención nutricional es una de las estrategias de mayor relevancia para mejorar desenlaces en ERAS, tanto en el periodo preoperatorio con la repleción nutricional y las intervenciones nutricionales como parte del proceso de prehabilitación y en el periodo postoperatorio con el inicio temprano de la vía oral. La doctora Sánchez de Costa Rica, presenta en un artículo de revisión, las barreras en la implementación de los aspectos relacionados con nutrición en la implementación de ERAS. Además, el artículo escrito por nuestro grupo de clínica Reina Sofía en Bogotá habla sobre las estrategias nutricionales prequirúrgicas, su implementación y resultados obtenidos, lo que permite conocer la forma como este gran programa se ha ido adoptando en América Latina.

ERAS representa una nueva manera de trabajar, es complejo en ocasiones aceptar el cambio, pero las estrategias utilizadas en el protocolo son sencillas y relativamente fáciles de implementar por lo que la voluntad de mejorar tiene un importante papel en el desarrollo y el éxito. La ciencia médica se ha convertido en una disciplina tan extensa como diversa haciendo que se tengan en cuenta muchos y muy variados elementos al momento de tratar un paciente. Este tipo de estrategias deberá ser considerado un estándar para la práctica quirúrgica por las instituciones hospitalarias de manera individual o como política de salud pública en donde se potenciarán las ventajas para los pacientes, el grupo de profesionales, las instituciones y el Sistema de Salud.

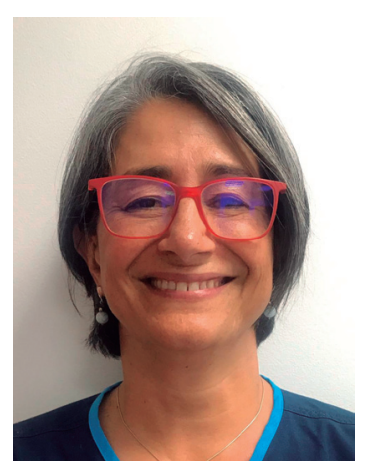

Ángela Navas Camacho, MD. Cirujana General. Médica Grupo de Soporte Nutricional Clínica Reina Sofía. Grupo ERAS Cirugía Colorrectal Clínica Reina Sofía (Centro de Excelencia ERAS). Fue Editora Asociada de la Revista de Nutrición Clínica y Metabolismo durante los primeros dos años de la Revista (2018-2019). Agradecemos su labor y el constante apoyo que ha brindado.

\section{Referencias bibliográficas}

1. Weiser TG, Haynes AB, Molina G, Lipsitz SR, Esquivel MM, Uribe-Leitz T, et al. Estimate of the global volume of surgery in 2012: an assessment supporting improved health outcomes. Lancet. 2015;385:S11. 
2. Organización Panamericana de la Salud, Organización Mundial de la Salud. Guía clínica para atención primaria a las personas adultas mayores. Promoción de salud y envejecimiento activo. Serie de materiales de capacitación. Número 1. 2002;10. [Internet] Consultado el: 20 enero de 2020. Disponible en: http://www.sld.cu/galerias/pdf/sitios/gericuba/introduccion.pdf
3. Goeree R, Diaby V. Introduction to health economics and decision-making: Is economics relevant for the frontline clinician? Best Pract Res Clin Gastroenterol. 2013;27(6):831-44. doi.org/10.1016/j.bpg.2013.08.016

4. Enhanced recovery after surgery, ERAS. [Internet] (Consultado el 3 de enero 2020). Disponible en: https:// erassociety.org/ 